\title{
Risk factor assessment of endoscopically removed malignant colorectal polyps
}

\author{
P Netzer, C Forster, R Biral, C Ruchti, J Neuweiler, E Stauffer, R Schönegg, C Maurer,
} J Hüsler, F Halter, A Schmassmann

\begin{abstract}
Background-Malignant colorectal polyps are defined as endoscopically removed polyps with cancerous tissue which has invaded the submucosa. Various histological criteria exist for managing these patients.

Aims-To determine the significance of histological findings of patients with malignant polyps.

Methods-Five pathologists reviewed the specimens of 85 patients initially diagnosed with malignant polyps. High risk malignant polyps were defined as having one of the following: incomplete polypectomy, a margin not clearly cancer-free, lymphatic or venous invasion, or grade III carcinoma. Adverse outcome was defined as residual cancer in a resection specimen and local or metastatic recurrence in the follow up period (mean 67 months).

Results-Malignant polyps were confirmed in 70 cases. In the 32 low risk malignant polyps, no adverse outcomes occurred; 16 $(42 \%)$ of the 38 patients with high risk polyps had adverse outcomes $(p<0.001)$. Independent adverse risk factors were incomplete polypectomy and a resected margin not clearly cancer-free; all other risk factors were only associated with adverse outcome when in combination.

Conclusion-As no patients with low risk malignant polyps had adverse outcomes, polypectomy alone seems sufficient for these cases. In the high risk group, surgery is recommended when either of the two independent risk factors, incomplete polypectomy or a resection margin not clearly cancer-free, is present or if there is a combination of other risk factors. As lymphatic or venous invasion or grade III cancer did not have an adverse outcome when the sole risk factor, operations in such cases should be individually assessed on the basis of surgical risk.

(Gut 1998;43:669-674)
\end{abstract}

Keywords: malignant polyps; colon cancer; colonoscopy; polypectomy; histology

Kantonsspital, St

Gallen, Switzerland

J Neuweiler

R Schönegg

Correspondence to: Dr A Schmassmann Gastrointestinal Unit, Inselspital, University of Berne, 3010 Berne,

Switzerland.

Accepted for publication 4 June 1998

Malignant colorectal polyps are defined as endoscopically removed adenomatous polyps containing cancerous tissue which has invaded the submucosa. ${ }^{1}$ Such malignant polyps are found in $0.2-9 \%$ of endoscopically removed adenomatous polyps, and in $9-11 \%$ if surgical series are included..$^{2-7}$ In recent years, polypectomy alone has been believed to be sufficient if certain histological criteria were met. ${ }^{128-10}$ Although an incomplete polypectomy or a margin of resection which is not clearly cancerfree are generally accepted to be high risk factors for an adverse outcome, other histological risk factors are still under debate. ${ }^{12} 5_{8-19}$ Cooper and colleatues ${ }^{9}$ recently suggested that a free resection margin greater than $1 \mathrm{~mm}$, no lymphatic or venous invasion, and poorly differentiated cancer (grade III) are risk factors, whereas Volk and colleagues ${ }^{8}$ only considered a free resection margin greater than 2 $\mathrm{mm}$ and poorly differentiated cancer to be risk factors. Other investigators have also taken into account the so called Haggitt level or have suggested that sessile polyps are a high risk factor for adverse outcome. ${ }^{11} 1314$

In a previous small study at another hospital, we were able to distinguish between low and high risk polyps on the basis of only two criteria, margin status and grading of invasive cancer as proposed by Volk et al. ${ }^{810}$ As that study was small and based only on the primary pathology record, we designed this study of clinical-histological re-evalution of patients with endoscopically removed malignant polyps to determine the efficacy of the various histological risk factors. Special attention was given to venous and lymphatic invasion, which is frequently a controversial topic in the literature. ${ }^{5-10}$

\section{Methods}

We reviewed the clinical records and specimens of 85 consecutive patients with an initial diagnosis of malignant polyps from 1980 to 1995 . Criteria for inclusion were the following: the polyp was initially removed endoscopically; the polyp contained an adenocarcinoma with invasion into the submucosa, a so called malignant polyp $^{17}$; and the availability of the patient's medical records and the histological slides of the malignant polyps. Excluded were patients with known synchronous adenocarcinoma, familial adenomatous polyposis, or idiopathic inflammatory bowel disease. The follow up data were collected during the clinical check ups and from a written survey of patients, their relatives and physicians. In addition, the patients/relatives were also interviewed by telephone as were, when necessary, their primary death certificates and autopsy reports.

The following endoscopic findings were assessed: polyp location, polyp size, polyp type, macroscopic entirety of local polyp resection, and complications after endoscopic polypectomy. 
Table 1 Characteristics of the 70 malignant polyps

\begin{tabular}{lcllll}
\hline Size (cm) & Number (\%) & Tubular (\%) & Tubulovillous (\%) & Villous (\%) & Cancer $(\%)$ \\
\hline$<1$ & $5(7)$ & $0(0)$ & $5(7)$ & $0(0)$ & $0(0)$ \\
$1-2$ & $37(53)$ & $5(7)$ & $25(36)$ & $1(1)$ & $6(9)$ \\
$>2$ & $23(33)$ & $2(3)$ & $16(23)$ & $0(0)$ & $5(7)$ \\
$>3$ & $5(7)$ & $1(1)$ & $4(6)$ & $0(0)$ & $0(0)$ \\
Total & $70(100)$ & $8(11)$ & $50(72)$ & $1(1)$ & $11(16)$ \\
\hline
\end{tabular}

* Six were classified as polypoid adenocarcinoma and five had an incomplete or piecemeal polypectomy, therefore only adenocarcinoma could reliably be diagnosed.

In patients with ensuing surgical resection, the following parameters were assessed: tumour tissue remaining in the resected bowel wall, regional lymph node metastases, and postoperative morbidity and mortality.

All slides were reviewed, without knowledge of the clinical outcome, by five pathologists experienced in gastrointestinal pathology; three came from our university hospital and two from the teaching hospital of St Gallen. The histological diagnoses of the two institutes were finally compared. In cases with differing diagnoses the findings were classified as uncertain, as they were when both diagnosed uncertain findings. ${ }^{8}$

Histological assessment included confirmation of the presence of invasive cancer and typing of tubular, tubulovillous, or villous adenomas according to standard histopathological criteria. The material was routinely processed for histopathological diagnosis: tissue was fixed in $4-10 \%$ formalin, sliced, totally embedded, and stained with haematoxylin and eosin. Additional staining to show vascular invasion was performed in 15 cases (Weigert's elasticvan Gieson and PAS). ${ }^{51620}$

Assessment was made of: (1) tumour status according to the WHO classification ${ }^{21}$; (2) the margin of the resected polyp: free or not free of cancer or uncertain (which included not assessable); the cancer-free distance was also measured; (3) histological grading of I-III (well, moderately, poorly differentiated); and (4) invasion of lymphatic and/or venous vessels. ${ }^{20}$

According to the presence or absence of unfavourable histological signs, polyps were classified as high or low risk. High risk malignant polyps were defined as having: tumour invasion in the margin or an uncertain margin; poor differentiation (grade III); or invasion of lymphatic or venous vessels. Low risk malignant polyps did not have any of these three histological signs.

Adverse outcome after polypectomy was defined as residual cancer found in the bowel wall or lymph nodes at the ensuing resection or the local recurrence of cancer or metastatic adenocarcinoma found at follow up. ${ }^{8-10}$

All statistical analyses were performed by the Department of Mathematical Statistics, University of Berne, which used the statistical software StatXact 3 for Windows (Cytel Software Corporation Cambridge, Massachusetts, USA). Unconditional exact test procedures for comparison of two proportions were used to compare the risk groups. ${ }^{22-24}$ Probability values of $\mathrm{p}<0.05$ (one sided) were regarded as significant. In addition, the odds ratios and two sided exact $95 \%$ confidence intervals (CI) were calculated (when the p value is between $2.5 \%$ and $5 \%$ the $95 \%$ CI includes 1$).^{22-24}$

\section{Results}

Of the initial 85 malignant polyps, 70 were included in the study. Reasons for exclusion were: 12 non-confirmations of cancerous invasion of the submucosa (carcinoma in situ); one possible ulcerative colitis; one synchronous lung cancer (possible metastatic disease); and in one case the original histology specimens were unavailable.

None of the 12 patients with carcinomas in situ had further problems from these polyps in the follow up period. However, one of the three patients subsequently reclassified as not having a malignant polyp but who submitted to resection died from postoperative complications. Although no cancerous tissue was found in his resected bowel wall, adenomatous tissue with severe dysplasia was found.

The median age was 69.5 years (range 43-88) and the sex ratio of females to males was $1: 2.1$. In the follow up period, four patients died within four months of diagnosis. Three died as a result of the ensuing colon resection. One, who also had a malignant lymphoma, died of pneumonia. For the other patients, the mean follow up period was 67 months (range 30-189).

Table 1 shows the histological characteristics of the 70 malignant polyps. The malignant polyps were localised as follows: 16 in the rectum $(22.8 \%), 52$ in the sigmoid colon $(74.4 \%)$, and two in the descending colon $(2.8 \%)$. Of the $51(73 \%)$ patients with pendunculated malignant polyps, six $(8.5 \%)$ had adverse outcomes, whereas of the $19(27 \%)$ patients with sessile (or not clearly classifiable) ones, 10 (53\%) had adverse outcomes. There was a significant difference between the pendunculated and sessile polyp groups regarding outcome (table 2). However, all the malignant polyps of patients

Table 2 Comparison of various risk factors with outcome

\begin{tabular}{|c|c|c|c|c|c|}
\hline Groups & Risk factors $(N)$ & $N$ & Adverse outcome & Odds ratio $(95 \% \mathrm{CI})$ & $p$ Value \\
\hline Polyp shape & †Sessile $v$ pendunculated & $19 v 51$ & $10 v 9$ & $8.3(2.1$ to 34.8$)$ & $<0.001$ \\
\hline Polypectomy & Incomplete $v$ complete & $8 v 62$ & $6 v 10$ & $15.6(2.2$ to 169$)$ & 0.001 \\
\hline${ }^{\star}$ Margin of resection & Not cancer-free $v$ cancer-free & $24 v 38$ & $9 v 1$ & 20.2 (2.6 to 998$)$ & $<0.001$ \\
\hline${ }^{\star}$ Lymphatic invasion & $\dagger$ Present $v$ not present & $6 v 56$ & $4 v 6$ & $16.7(1.8$ to 204$)$ & 0.005 \\
\hline *Venous invasion & †Present $v$ not present & $5 v 57$ & $2 v 8$ & $4.1(0.3$ to 40.7$)$ & 0.18 \\
\hline$\star$ Grade III cancer & †Present $v$ not present & $5 v 57$ & $2 v 57$ & $4.1(0.3$ to 40.7$)$ & 0.18 \\
\hline$\star$ Polypoid cancer & †Present $v$ not present & $6 v 56$ & $3 v 7$ & $7(0.7$ to 60.6$)$ & 0.048 \\
\hline Risk categories & High risk $v$ low risk & $38 v 32$ & $16 v 0$ & Infinite ( 5 to infinite) & $<0.001$ \\
\hline
\end{tabular}

The odds ratio for an adverse outcome is given regardless of the presence or absence of various risk factors.

${ }^{\star}$ Eight of the 70 malignant polyps had incomplete or piecemeal resection; as their histological features could not reliably be determined, they were excluded from this analysis.

†These risk factors had only an adverse outcome in combination with other risk factors. 
Table 3 Treatment, risk categories and adverse outcome of the 70 malignant polyps

\begin{tabular}{llllllll}
\hline Treatment group & Number (\%) & Low risk & $\begin{array}{c}\text { Adverse } \\
\text { outcome }\end{array}$ & High risk & $\begin{array}{c}\text { Adverse } \\
\text { outcome }\end{array}$ & $\begin{array}{l}\text { Death due to } \\
\text { malignant polyp }\end{array}$ & $\begin{array}{l}\text { Treatment } \\
\text { mortality }\end{array}$ \\
\hline Polypectomy only (56\%) & $39(100)$ & $25(64)$ & 0 & $14(36)$ & $2(5)$ & $1(3)$ & 0 \\
Polypectomy + surgery (44\%) & $31(100)$ & $7(23)$ & 0 & $24(77)$ & $14(45)$ & $2(6)$ & $2(6)$ \\
Total & $70(100)$ & $32(46)$ & 0 & $38(54)$ & $16(23)$ & $3(4)$ & $2(3)$ \\
\hline
\end{tabular}

Percentage is given in brackets; adverse outcome defined as residual cancer in a resection specimen and local or metastatic recurrence during the follow up period.

High risk malignant polyps were when the following items were noted: incomplete polypectomy, not clearly cancer-free margin of resection, angiolymphatic invasion or grade III carcinoma. All others were classified as low risk.

with adverse outcomes had an unfavourable histology.

Table 3 summarises the treatment and adverse outcomes of the patients with malignant polyps. Uncomplicated polypectomy was performed in all patients. Polypectomy alone was performed on $39(56 \%)$ patients with malignant polyps; two (5\%) had an adverse outcome. There were no adverse outcomes for the 25 patients $(64 \%)$ with low risk polyps who underwent polypectomy alone. The remaining 14 patients $(36 \%)$ who were considered to have high risk malignant polyps were not operated on for the following reasons: refusal-two; severe contraindication for operation-one; negative second endoscopy-six; and initially misassessed as low risk-five. Of these 14 patients, two had adverse outcomes; although both had initially had a doubtful margin, the resection site control biopsy samples were negative. However, local recurrence was found in both patients in the follow up period. One was operated on and remains in good health three years later. The other developed metastases and died.

A total of 31 (44\%) patients with confirmed malignant polyps subsequently underwent surgery. Of the seven $(22.6 \%$ of surgical patients) at low risk, none had cancer in the resected bowel wall, and all of them had a favourable outcome at follow up. Of the $24(77.4 \%)$ at high risk, $14(45.1 \%)$ had an adverse outcome (summarised in table 5). Two (6.5\%) of the 31 patients died of postoperative complications; if the three surgical patients with carcinoma in situ who were initially diagnosed as having malignant polyps (see above) are included, then three $(8.8 \%)$ of the 34 surgical patients died: one from postoperative sepsis, one from mesenteric vessel thrombosis, and one from postoperative pneumonia. Death due to malignant polyps occurred in two of the surgical patients and both polyps had unfavourable histology. One patient had cancer in the resected bowel wall with negative lymph nodes, and developed distant metastases during follow up. The other had a negative bowel wall and negative local lymph nodes, but also developed distant metastases during follow up.
INCOMPLETE POLYPECTOMY

Eight $(11.4 \%)$ of the 70 patients with malignant polyps had an incomplete resection and/or a badly fragmented polypectomy, only allowing histological assessment of malignancy. Although no further histological risk factor analysis was possible in this group, it was included in the follow up. Seven underwent surgery, six had residual cancer, and one developed a local recurrence after two years. He died of a lung embolism 10 days after the second operation. The patient who did not have surgery had a second snare resection of the polyp stalk, which did not contain cancerous tissue. He remains well five years later. For adverse outcome there was a significant difference between this group and that with complete polypectomy (table 2).

\section{COMPLETE POLYPECTOMY}

Of the remaining $62(100 \%)$ polyps with a detailed histological analysis, $30(48.4 \%)$ were considered high risk of which 10 (16.1\%) had an adverse outcome. No adverse outcome was observed in any patients with low risk malignant polyps. Table 4 summarises the histological risk factors of these 62 malignant polyps.

\section{RESECTION MARGIN}

A high interobserver correlation was found for assessment of the margin status. Twenty four of the 62 polyps $(38.7 \%)$ had a margin which was not cancer-free or was doubtful. Nine of the 24 $(38 \%)$ whose margin was not cancer-free, had adverse outcomes, of which four also had additional risk factors (table 5). Patients whose margins were not cancer-free or were doubtful had significantly more adverse outcomes compared with the others (table 2). In addition, only one malignant polyp with a cancer-free resection margin (cancer margin distance more than $2 \mathrm{~mm}$ ) had an adverse outcome; it did, however, have other risk factors. Of the 32 malignant polyps with a cancer-free resection margin and no other risk factors, no adverse outcomes were observed; this was independent of the width of the resection margin even though that of nine polyps $(28.1 \%)$ was less than $2 \mathrm{~mm}$.

Table 4 Risk factors and adverse outcome of the $62^{\star}$ malignant polyps with a detailed histological examination

\begin{tabular}{|c|c|c|c|c|c|c|}
\hline \multirow[b]{2}{*}{ Risk factors } & \multicolumn{2}{|c|}{ As sole risk factor } & \multicolumn{2}{|c|}{ With other risk factors } & \multicolumn{2}{|c|}{ Total } \\
\hline & $n$ & Adverse outcome (\%) & $n$ & Adverse outcome (\%) & $n$ & Adverse outcome (\%) \\
\hline Margin of resection & 19 & $5(26)$ & 5 & $4(80)$ & 24 & $9(38)$ \\
\hline Grade III & 1 & $0(0)$ & 4 & $2(50)$ & 5 & $2(40)$ \\
\hline Lymphatic invasion & 0 & $0(0)$ & 6 & $4(67)$ & 6 & $4(66)$ \\
\hline Venous invasion & 2 & $0(0)$ & 3 & $2(67)$ & 5 & $2(40)$ \\
\hline
\end{tabular}

*Of the 70 malignant polyps, the eight malignant polyps with incomplete or piecemeal polypectomy were excluded. Adverse outcome was defined as residual cancer in a resection specimen and local or metastatic recurrence during the follow up period. 
Table 5 Adverse outcome of the 16 malignant polyps

\begin{tabular}{|c|c|c|c|c|c|c|c|c|c|c|c|c|c|}
\hline Case & Sex & Site & $\begin{array}{l}\text { Size } \\
(\mathrm{cm})\end{array}$ & $\begin{array}{l}\text { Pendunc- } \\
\text { ulated }\end{array}$ & $\begin{array}{l}\text { Polypoid } \\
\text { cancer }\end{array}$ & $\begin{array}{l}\text { Margin not } \\
\text { cancer-free }\end{array}$ & $\begin{array}{l}\text { Grade } \\
\text { III }\end{array}$ & $\begin{array}{l}\text { Lymph } \\
\text { invasion }\end{array}$ & $\begin{array}{l}\text { Venous } \\
\text { invasion }\end{array}$ & $\begin{array}{l}\text { High } \\
\text { risk }\end{array}$ & $O P$ & $\begin{array}{l}\text { Adverse outcome } \\
\left({ }^{\star} \text { follow up) }\right.\end{array}$ & Comment \\
\hline 1 & M & Sigmoid & $>3$ & No & No & Yes & No & No & No & Yes & No & ${ }^{\star}$ Local recurrence & $\begin{array}{l}\text { Follow up } \\
\text { unremarkable }\end{array}$ \\
\hline 2 & $\mathrm{~F}$ & Rectum & $1-2$ & No & No & Yes & No & No & No & Yes & No & $\begin{array}{l}{ }^{\star} \text { Local recurrence }+ \\
\text { metastasis }\end{array}$ & MP death \\
\hline 3 & $\mathrm{~F}$ & Rectum & $<1$ & No & No & Yes & No & No & No & Yes & Yes & Residual carcinoma & \\
\hline 4 & $M$ & Rectum & $2-3$ & No & No & Yes & No & No & No & Yes & Yes & Residual carcinoma & \\
\hline 5 & $M$ & Sigmoid & $2-3$ & No & No & Yes & No & No & No & Yes & Yes & $\begin{array}{l}\text { Residual carcinoma } \\
+\star{ }^{\star} \text { local recurrence }\end{array}$ & $\begin{array}{l}\text { Postoperative } \\
\text { death }\end{array}$ \\
\hline 6 & M & Rectum & $1-2$ & No & No & Yes & No & No & No & Yes & Yes & Residual carcinoma & \\
\hline 7 & $\mathrm{~F}$ & Sigmoid & $2-3$ & Yes & No & Yes & No & No & No & Yes & Yes & Residual carcinoma & \\
\hline 8 & $\mathrm{M}$ & Sigmoid & $2-3$ & Yes & No & Yes & No & No & No & Yes & Yes & Residual carcinoma & \\
\hline 9 & M & Sigmoid & $1-2$ & No & Yes & Yes & Yes & Yes & Yes & Yes & Yes & Residual carcinoma & \\
\hline 10 & $M$ & Sigmoid & $2-3$ & No & No & Yes & Yes & No & No & Yes & Yes & $\begin{array}{l}\text { Residual carcinoma } \\
+{ }^{\star} \text { metastasis }\end{array}$ & MP death \\
\hline 11 & M & Sigmoid & $2-3$ & No & Yes & Yes & No & No & No & Yes & Yes & Residual carcinoma & $\begin{array}{l}\text { Postoperative } \\
\text { death }\end{array}$ \\
\hline 12 & $M$ & Sigmoid & $>3$ & Yes & No & Yes & No & No & No & Yes & Yes & Residual carcinoma & \\
\hline 13 & $M$ & Sigmoid & $1-2$ & Yes & No & Yes & No & No & No & Yes & Yes & $\begin{array}{l}\text { Residual carcinoma } \\
+ \text { positive lymph } \\
\text { node }\end{array}$ & \\
\hline 14 & $M$ & Sigmoid & $2-3$ & Yes & Yes & No & No & Yes & Yes & Yes & Yes & Positive lymph node & \\
\hline 15 & M & Rectum & $1-2$ & No & No & Yes & No & Yes & No & Yes & Yes & Residual carcinoma & \\
\hline 16 & M & Sigmoid & $2-3$ & Yes & No & Yes & No & Yes & No & Yes & Yes & ${ }^{\star}$ Metastasis & MP death \\
\hline
\end{tabular}

High risk malignant polyps were when the following items were noted: incomplete polypectomy, not clearly cancer-free margin of resection, angiolymphactic invasion, or grade III carcinoma.

VESSEL INVASION

Nine of the 62 polyps $(14.5 \%)$ had vessel invasion: four had lymphatic invasion, three venous, and two both. Lymphatic invasion was found in six polyps of which four had an adverse outcome, but all had additional risk factors (table 5). There was a significant difference in outcome between the groups with and without lymphatic invasion (table 2). Venous infiltration was found in five patients, two of whom had an adverse outcome, but both had additional risk factors (table 5). There was no statistically significant difference in outcome between the groups with and without venous infiltration (table 2).

In addition to these nine cases, five others were uncertain ( $8 \%$ of all 62 polyps): four had possible lymphatic vessel invasion and in one of them the vessel type was also uncertain. One of these patients had an adverse outcome, but he also did not have a cancer-free resection margin.

POORLY DIFFERENTIATED CARCINOMA (GRADE III) Five $(8 \%)$ were poorly differentiated, of which two had an adverse outcome, but both had additional risk factors (table 5). There was no statistically significant difference in outcome between the groups with well or moderately and poorly differentiated carcinoma (table 2).

The two pathological institutes differed as to whether the diagnosis was grade II or III in five $(8 \%)$ cases. They were finally classified as grade II. An adverse outcome was found in two of these five, but both had additional risk factors (one without a cancer-free margin, the other with lymphatic and venous vessel invasion).

POLYPOID CARCINOMA

Six patients $(9.7 \%)$ had a polypoid cancer, three of which had an adverse outcome; all had additional risk factors (table 5). Two had residual cancer in the bowel wall and one had a tumour-free bowel wall but local lymph node metastases. None died from the malignant polyp, but one died of postoperative sepsis.
There was borderline significant difference in outcome between the groups with and without polypoid carcinoma (table 2 ).

OVERALL RATE OF ADVERSE OUTCOME

When, in addition to the 62 malignant polyps with complete polypectomy, the eight with incomplete polypectomy are also considered, the overall rate of adverse outcome of all 70 malignant polyps was $22.8 \%$ (16 polyps) or $42.1 \%$ of the 38 high risk malignant polyps (table 5). None of the low risk malignant polyps had an adverse outcome. There was a highly significant difference in outcome between the groups with low and high risk malignant polyps (table 2).

\section{Discussion}

As most colorectal cancers arise from benign adenomas via the adenoma-carcinoma sequence, it is not surprising to find an early invasive adenocarcinoma in endoscopically removed polyps (the so called malignant polyp). ${ }^{125-27}$ There are several histological features which have been suggested to be risk factors for an adverse outcome. Although incomplete polypectomy and a cancer containing resection margin are well accepted, the other risk factors are still under debate. The findings of our study, in combination with previously published results, may help the clinician to balance more accurately the relative risks of surgery against those of death from metastatic cancer.

In our study there was an overall adverse outcome in 16 of our 70 patients with malignant polyps $(22.8 \%)$. All 16 belonged to the group of 38 high risk malignant polyps, which were defined as having a margin which was not cancer-free and/or poorly differentiated cancer (grade III) and/or vessel invasion. ${ }^{8} 9$

The most important adverse risk factor was incomplete polyp resection. This was found in eight polyps $(11.4 \%)$, six of which $(75 \%)$ had an adverse outcome; this emphasises the 
importance to the histologist of the gastroenterologist's polypectomy report, as it is well accepted that such patients need surgery. ${ }^{2} 891215$ As the detailed histological features could not be reliably determined, we excluded incompletely removed polyps from further histological risk assessment.

Of the remaining 62 polyps, the most common feature of high risk malignant polyps was a resection margin which was not clearly cancer-free; this was found in $24(38.7 \%)$ of these polyps. An adverse outcome was found in nine of these and in five of them such a resection margin had been the sole risk factor. We found these margins, together with incomplete polypectomy, to be the only independent risk factors. ${ }^{7}$ However, residual carcinoma was not found in all of those cases sent for resection; it was only present in $40 \%$. In the remaining cases, diathermy may have destroyed any residual tumour in the polyp base. ${ }^{215} 16$ There were 10 patients whose margin was not clearly cancer-free but who had no ensuing operation; two $(20 \%)$ had an adverse outcome even though they had both had a second endoscopy after the diagnosis of their malignant polyp which did not show any evidence of residual cancer. Performing a second endoscopy in those patients whose resection margin is not clearly cancer-free, does not seem to be useful. The importance of the resection margin is also supported by the finding that only one of all the 16 cases with adverse outcome had a clearly cancer-free margin (greater than $2 \mathrm{~mm}$ ).

Although surgery for patients with a margin which is not clearly cancer-free is well accepted, the requisite distance from cancer to margin is still under discussion. ${ }^{28911} 121516$ None of our patients with a clearly free resection margin and no other risk factors had an adverse outcome, even though we found that $28 \%$ of polyps had a margin-cancer distance of less than $2 \mathrm{~mm}$. The rate of detecting high risk malignant polyps would probably be too high if a measurable margin of $2 \mathrm{~mm}$ or more were insisted on. ${ }^{2} 3812151628$ The pathologist's declaration of a clearly cancer-free margin of excision, independent of the distance, together with the endoscopist's opinion of total polyp excision seems sufficient for further management. ${ }^{121516}$

The diagnosis of angiolymphatic vessel invasion is not easy and the interobserver correlation is reported as only fair. ${ }^{8}{ }^{9}$ For our polyps the diagnosis was definite in nine $(14.5 \%)$ and uncertain in five $(8 \%)$. Of these five, only one, which had additional risk factors, had an adverse outcome; therefore uncertain vessel invasion can hardly be classed as an independent risk factor. ${ }^{7916}$ In fact, only Cooper et al found it to be so. ${ }^{9}$

When venous invasion (found in $8 \%$ ) was the only risk factor, as occurred in three cases ( $5 \%)$, no adverse outcomes were noted. Venous infiltration was also infrequently diagnosed in other studies, ${ }^{2} 91129$ excepting that of Geraghty et $a l .{ }^{30}$ In most other studies, except that of Muller et $a l,{ }^{5}$ venous invasion was not an independent risk factor either, and is therefore hardly justified as the only criterion for surgical intervention. ${ }^{589121430-32}$
Lymphatic invasion was found in six of our malignant polyps $(9.7 \%)$ and was significantly more often associated with an adverse outcome than those without. However, all of our lymphatic invasion cases with adverse outcomes also had an additional risk factor, as has been reported in almost all studies, excepting that of Cooper et al. ${ }^{158911121530}$ The presence of lymphatic invasion alone as a risk factor is, as reported, a rare event and did not occur in our study. In the small number of cases with lymphatic invasion, in the absence of other unfavourable features, we believe that the indication for an operation should be individually assessed on the basis of surgical risk. ${ }^{16}$

We found lymph node metastases in two patients at ensuing bowel resection $(6.4 \%$ of all surgically treated malignant polyps), and both polyps had been assessed as high risk. In the literature, the incidence of lymph node metastases varies greatly from $0 \%$ to over $20 \%$, with an estimated average rate of $8-10 \%$ when surgical series are also included. ${ }^{2} 469142933$ However, when only patients with low risk malignant polyps were considered, the risk for nodal disease is probably less than $1 \%$, which is lower than the average risk of surgical bowel resection. $^{2} 89153134-36$

Although a poorly differentiated carcinoma is a well accepted risk factor associated with an increased risk of early lymph node metastases and haematogenous spread, all such cases with adverse outcomes had additional risk factors, as previously reported. ${ }^{2689153034}$ In addition, they are rare and are reported as comprising less than $10 \%$ of malignant polyps in most studies. ${ }^{915} 31$ We only found five (8\%) malignant polyps with a poorly differentiated carcinoma. In the one which had a poorly differentiated carcinoma as the sole risk factor, no adverse outcome was noted. ${ }^{2}{ }^{9}$ However, in five additional cases the two pathology institutes differed as to whether the initial diagnosis was grade II or III. No patients with only uncertain grade III cancer as the sole risk factor had an adverse outcome. When it occurs as the sole risk factor, surgery should be individually assessed on the basis of surgical risk.

In diagnosing both vessel invasion and grade III carcinoma, the interobserver correlation was only fair. ${ }^{9}$ The criteria used for these diagnoses were identical in both institutes and are used in their daily routine. On the basis of our findings, a second opinion from a pathologist experienced in gastrointestinal diagnosis should be requested in cases where the only risk factor is vessel invasion or a grade III carcinoma.

Polypoid cancer, defined as when the entire polyp epithelium is cancerous, was found in six $(9.7 \%)$ patients. ${ }^{9}{ }^{15}$ Five of them were high risk, and in three cancerous tissue was found at the ensuing operation. However, no local recurrence or metastatic disease was noted during the follow up. The majority of authors, as we did, treated polypoid cancers the same as malignant polyps because, in various studies, they were no more aggressive. ${ }^{2} 7911152934$

For polypectomy, pedunculated and sessile malignant lesions were treated identically, in accordance with other (but not all) investiga- 
tors' recommendations. ${ }^{7} 9111314$ However, sessile malignant polyps had unfavourable histology more often and were also significantly more frequently associated with adverse outcomes than pendunculated ones. Nevertheless, as low risk malignant polyps, independent of their appearance, had no adverse outcomes, we support the suggestion that, when feasible, polypectomy is sufficient for all low risk malignant polyps. ${ }^{2} 8915$

To manage patients with malignant polyps further, the comparative risk of surgery needs to be evaluated in addition to that of developing metastatic cancer. In comparison to surgery, endoscopic polypectomy is a procedure with a low overall complication rate of about $3 \%$, and a mortality rate of less than $0.1 \% .^{2}$ In our patients, no severe complications resulted from the endoscopic procedure. Of the 34 patients who had an ensuing operation, including the three malignant polyps that were reclassified as non-malignant at histological revision, six $(17.6 \%)$ had relevant complications and three $(8.8 \%)$ died after surgery, including one of those thought on review to only have "carcinoma in situ" and so was excluded from the main analysis. This complication rate is consistent with that reported in the literature, giving a mortality rate of up to $3 \%$ for patients less than 70 years old which increases to $12 \%$ in patients over $70 .^{2} 313536$

One should also consider that, in spite of an ensuing operation after polypectomy and even when no residual carcinoma or positive lymph nodes were found, a local recurrence or metastatic disease can occur. In our study, three patients developed a local recurrence or distant metastases postoperatively, one of whom had not had residual carcinoma or metastases at surgery. Similar experiences have been reported elsewhere. ${ }^{91529}$ It seems that, in a low percentage of patients, there is fatal outcome regardless of the form of treatment. ${ }^{9}$

In conclusion, the most important and the only independent risk factors for adverse outcome in patients with malignant polyps were incomplete polypectomy and a resection margin that was histologically not clearly cancer-free. Consequently, all patients fit for operation with such high risk malignant polyps, should be submitted to surgery. All other risk factors only had an adverse outcome when combined with others. In the small number of cases with one of these lesser risk factors in the absence of other unfavourable features, the indication for operation is questionable and should be individually assessed on the basis of surgical risk. As no patients with low risk malignant polyps had an adverse outcome, polypectomy seems to be sufficient for malignant polyps with low risk histological features.

The authors are grateful for the language assistance of Oonagh Heron and the secretarial assistance of Maya Mettler. The study was performed at the Inselspital, University Hospital of Berne, in collaboration with the Institute of Pathology, Kantonsspital, St Gallen, and presented at the 97th DDW, Washington, DC, 1997 and also published in abstract form (Gastroenterology 1997;112:627A).

1 Bond JH. Polyp guideline: diagnosis, treatment and surveillance for patients with nonfamilial colorectal polyps. Ann Intern Med 1993;119:836-43.
2 Hackelsberger A, Frühmorgen P, Weiler H, et al. Endoscopic polypectomy and management of colorectal adenomas polypectomy and management of colorectal ade
with invasive carcinoma. Endoscopy 1995;27:153-8.

3 Hermanek P, Frühmorgen P, Guggenmoos-Holzmann I, et al. The malignant potential of colorectal polyps. Endoscopy 1983;15:16-20

4 Muto T, Sawada T, Sugihara K. Treatment of carcinoma in adenomas. World $\mathcal{F}$ Surg 1991;15:35-40.

5 Muller S, Chesner IM, Egan MJ, et al. Significance of venous and lymphatic invasion in malignant polyps of the colon and rectum. Gut 1989;30:1385-91.

6 Coverlizza S, Risio M, Ferrar A, et al. Colorectal adenomas containing invasive carcinoma. Cancer 1989;64:1937-47.

7 Cranley JP, Petras RE, Carey WD, et al. When is endoscopic polypectomy adequate therapy for colonic polyps containng invasive carcinoma? Gastroenterology 1986;91:419-27.

8 Volk EE, Goldblum JR, Petras RE, et al. Management and outcome of patients with invasive carcinoma arising in colorectal polyps. Gastroenterology 1995;109:1801-7.

9 Cooper HS, Deppisch LM, Gourley WK, et al. Endoscopically removed malignant colorectal polyps: clinicopathologic correlations. Gastroenterology 1995;108:1657-65.

10 Netzer P, Binek J, Hammer B, et al. Significance of histologic criteria for the management of patients with malignant colorectal polyps and polypectomy. Scand $\mathcal{f}$ Gastroenterol 1997;32:910-16.

11 Haggitt RC, Glotzbach RE, Soffer EE, et al. Prognostic factors in colorectal carcinomas arising in adenomas: implications for lesions removed by endoscopic polypectomy. Gastroenterology 1985;89:328-36.

12 Cunningham KN, Mills LR, Schuman BM, et al. Long-term prognosis of well-differentiated adenocarcinoma in endoscopically removed colorectal adenomas. Dig Dis Sci 1994; 39:2034-7.

13 Pollard CW, Nivatvongs S, Rojanasakul A, et al. The fate of patients following polypectomy alone for polyps containing invasive carcinoma. Dis Colon Rectum 1992;35:933-7.

14 Nivatvongs S, Rojanasakul A, Reiman HM, et al. The risk of lymph node metastasis in colorectal polyps with invasive lymph node metastasis in colorectal polyps with i

15 Morson BC, Whiteway JE, Jones EA, et al. Histopathology and prognosis of malignant colorectal polyps treated by endoscopic polypectomy. Gut 1984;25:437-44

16 Jass JR. Malignant colorectal polyps [editorial]. Gastroenterology 1995;109:2034-5.

17 Cooper HS. Surgical pathology of endoscopically removed malignant polyps of the colon and rectum. Am F Surg Pathol 1983;7:613-23.

18 Langer JC, Cohen Z, Taylor BR, et al. Management of patients with polyps containing malignancy removed by

colonoscopic polypectomy. Dis Colon Rectum 1984;27:6-9.
19 Richards WO, Webb WA, Morris SJ, et al. Patient management after endoscopic removal of the cancerous colon adenoma. Ann Surg 1987;205:665-72.

20 Morson BC, Dawson IM, Day DW, et al. Morson E Dawson's gastrointestinal pathology. 3rd edn. Oxford: Blackwell Scientific Publications, 1989

21 Hermanek P, Sobin LH. TNM classification of malignant tumors. 4th edn. Berlin: Springer, 1992.

22 Agresti A. Exact tests for small samples. In: Agresti A, editor. Categorical data analysis. New York: J Wiley and Sons, 1990:36-78.

23 Santer TJ, Snell MK. Small-sample confidence intervals for p1-p 2 and $\mathrm{p} 1 / \mathrm{p} 2$ in $2 \times 2$ contingency tables. $\mathcal{F}$ Am Stat Assoc 1980;75:386-94.

24 Berger RL, Boos DD. Values maximized over a confidence set for the nuisance parameter. F Am Stat Assoc 1998;89: set for the

25 Morson BC. The polyp-cancer sequence in the large bowel. Proc R Soc Med 1974;67:451-7.

26 Fearon ER, Vogelstein B. A genetic model for colorectal tumorigenesis. Cell 1990;61:759-67.

27 Winawer SJ, Zauber AG, Nah Ho MS, et al. Prevention of colorectal cancer by colonoscopic polypectomy. $N$ Engl $\mathcal{F}$ Med 1993;329:1977-81.

28 Colacchio TA, Forde KA, Scantlebury VP. Endoscopic polypectomy - inadequate treatment for invasive colorectal carcinoma. Ann Surg 1981;194:704-7.

29 Kyzer S, Begin LR, Gordon PH, Mitmaker B. The care of patients with colorectal polyps that contain invasive adenocarcinoma. Endoscopic polypectomy or colectomy? Cancer 1992;70:2044-50.

30 Geraghty JM, Williams CB, Talbot IC. Malignant colorectal polyps: venous invasion and successful treatment by endopolyps: venous invasion and succesful tr

31 Chantereau MJ, Faivre J, Boutron MC, et al. Epidemiology, Chantereau MJ, Faivre J, Boutron MC, et al. Epidemiology,
management and prognosis of malignant large bowel polyps within a defined population. Gut 1992;33:259-63.

32 Talbot IC, Ritchie S, Leighton M, et al. Invasion of veins by carcinoma of rectum: method of detection, histological features and significance. Histopathology 1981;5:141-63.

33 Minamoto T, Mai M, Ogino T, et al. Early invasive colorectal carcinomas metastatic to the lymph node with attention to their nonpolypoid development. Am $\mathcal{f}$ Gastroenterol 1993;88: 1035-9.

34 Christie JP. Polypectomy or colectomy? Management of 106 consecutively encountered colorectal polyps. Am Surg 1988;54:93-9.

35 Greenburg AG, Saik RP, Coyle JJ, et al. Mortality and gastrointestinal surgery in aged. Arch Surg 1981;116:788-91.

36 Fielding LP, Philips RK, Hittinger R. Factors influencing mortality after curative resection for large bowel cancer in elderly patients. Lancet 1989;i:595-7. 